\title{
Porosity Estimation from High Resolution CT SCAN Images of Rock Samples by Using Housfield Unit
}

\author{
Nguyen Lam Quoc Cuong, Nguyen Hong Minh, Hoang Manh Cuong, Phan Ngoc Quoc, \\ Ngo Hoang Van Anh, Nguyen Van Hieu
}

Analysis Laboratory Center-Vietnam Petroleum Institute, Ho Chi Minh City, Vietnam

Email: hieunv@vpi.pvn.vn

How to cite this paper: Cuong, N.L.Q., Minh, N.H., Cuong, H.M., Quoc, P.N., Van Anh, N.H. and Van Hieu, N. (2018) Porosity Estimation from High Resolution CT SCAN Images of Rock Samples by Using Housfield Unit. Open Journal of Geology, 8, 1019-1026.

https://doi.org/10.4236/ojg.2018.810061

Received: July 18, 2018

Accepted: September 23, 2018

Published: September 26, 2018

Copyright (c) 2018 by authors and Scientific Research Publishing Inc. This work is licensed under the Creative Commons Attribution International License (CC BY 4.0).

http://creativecommons.org/licenses/by/4.0/

\begin{abstract}
Computer Automated Tomography has been shown to be a valuable tool in production research because it provides a non-destructive method to identify and evaluate the internal structural characteristics of reservoir rock. In CT scan, Hounsfield Unit (HU) is proportional to the degree of X-ray attenuation by the tissue. The aim of the present study was to introduce the method to estimate porosity which is one of physical parameters of reservoir rock though HU data. In this study, an Image J software was used to extract Hounsfield Unit data and calibrate by standard material's density. This method provides the ability of using CT Scanner in advanced reservoir characterization and flow test experiments.
\end{abstract}

\section{Keywords}

CT Scan, Hounsfield Unit, Porosity

\section{Introduction}

Computer Tomography (CT scanning) was originally developed for the medical sector (Brooks and Di Chiro, 1975; Rutherford et al., 1976a; Dubal and Wiggli, 1977; Morgan, 1983) and has since found wide applications within the petroleum and other industries (Rutherford et al., 1976b; Vinegar, 1986; Wellington and Vinegar, 1987). Within the petroleum industry, CT scanning technology is used to study core samples from oil and gas reservoirs, with applications in both areas of core analysis and petrophysics as well as multiphase fluid flow. CT scanners offer researchers the capability of rapid, nondestructive visualization and analysis of the internal structure of core materials and experiments involv- 
ing core material [1]. CT scanners are used to provide images of sleeved and preserved core, and to identify and characterize fractures, in homogeneities, and zones of mud invasion thereby facilitating the selection of appropriate sampling intervals. Hounsfield Unit is found by Sir Godfrey Newbold Hounsfield-one of the principal engineers and developers of computed axial tomography (CAT, or CT scans).

With the powerful of this system, we decided to carry out CT scanning on 9 samples from variation wells at different basins in Vietnam such as Cuu Long basin, South Con Son basin, Song Hong basin to release the method to estimate porosity by Hounsfield units. The samples grouped as limestone, sandstone, fractured granite. These samples have Helium porosity ranges from $2.65 \%$ to $41.7 \%$.

\section{Theory and Calculation Process}

As described in previous references, during the CAT scanning process the attenuation of an X-ray beam is measured as it passes through a sample material. When a parallel monochromatic X-ray beam passes through a substance of uniform density and atomic number, it is attenuated in an exponential manner such that:

$$
I=I_{0} * \mathrm{e}^{-\mu x}
$$

where $I_{0}$ and $I$ represent the intensity of the X-ray beam before and after passing through the substance, $x$ is the thickness of the material and $\mu$ is defined as the linear attenuation coefficient. This formula was taken from NDT Resourse Center. Figure 1 illustrates the CT scanning process.

The linear attenuation coefficient $\mu$ is defined as the fractional decrease in $\mathrm{X}$-ray intensity per unit length of that material and is a function of the atomic number and bulk density of the material and the energy of the probing X-rays. Generally, the linear attenuation coefficient is normalized to that of a standard material (such as water or air) that means each material is assigned by a unchanged number that help to calculate another material number and is defined as the Hounsfield Units (HU) or CT number of the material:

$$
H U=\frac{\left(\mu_{\text {material }}-\mu_{\text {water }}\right)}{\left(\mu_{\text {water }}-\mu_{\text {air }}\right)} * 1000
$$

Within a single tomographic scan, the X-ray attenuation is measured for a multitude of different angles and a cross-sectional reconstructed image is generated which represents the X-ray attenuation (CT number) in specific voxels (volume elements) of the material in a plane perpendicular to the motion of the scan (as illustrated in Figure 2). In the tomographic image light areas represent high X-ray attenuation or CT number (high density or atomic number) and darker areas represent low X-ray attenuation or CT number (low density or atomic number) [2] [3] [4].

However, the issue is how to extract the Hounsfield Units of the single 
cross-sectional slice. Therefore, we figure out the Image J software to solve this issue [5] [6]. Image J is a public domain Java image processing program suitable to measure distances and angles, to calculate area and pixel value statistics of user-defined selections, provide density histograms and line profile plots and so on. The measurement process will follow the flow chart in Figure 2.

Porosity is defined as the ratio of void volume to total volume in a soil sample. The CT scanning process provides a description of the solid and void spaces shown in cross-sections through rock samples. The steps to calculate the porosity of a rock pictured in a CT scan image are:

1) Selecting the region of interest (ROI);

2) Calibrate density;

3) Extract HU values;

4) Statistics HU values;

5) Calculate porosity.

The image processing steps were completed for this research using the Image J image analysis software, developed by the National Institutes of Health. These steps are described in detail in the following.

Selecting the region of interest removes much of "noise" and reduces "error" (isolated high intensity pixels) of digital image during CT scanning (Figure 3) [6] [7].

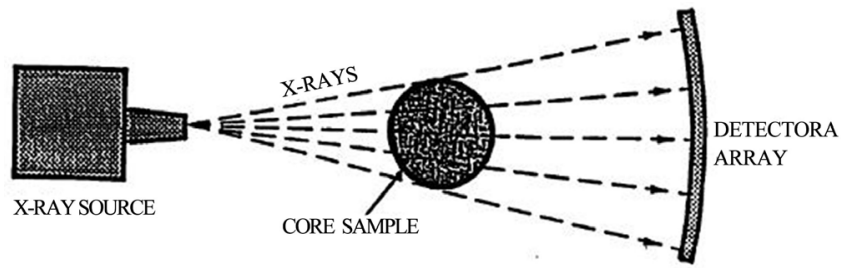

Figure 1. X-ray attenuation measurement process.

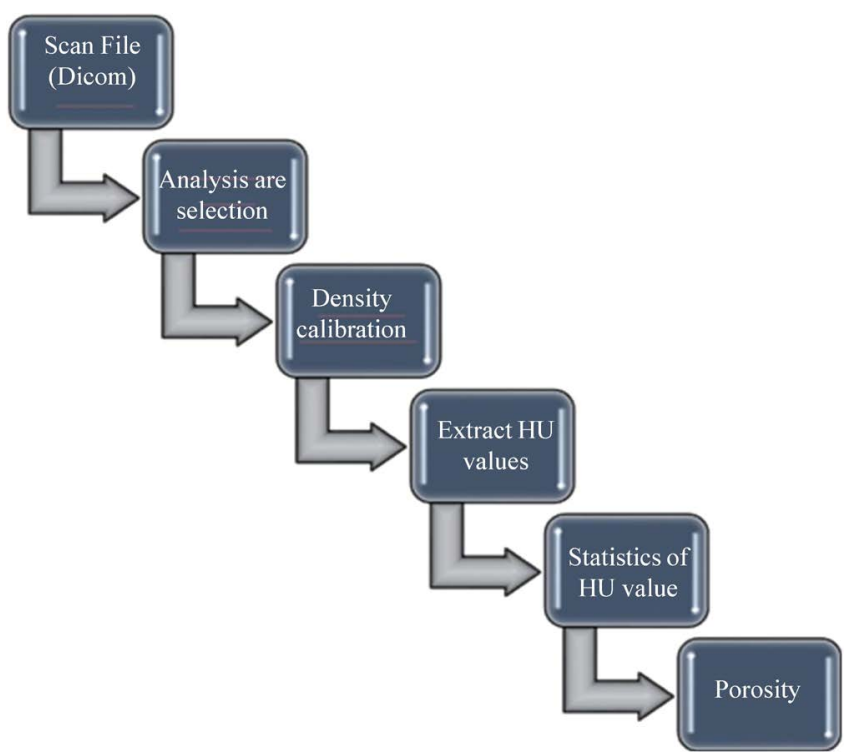

Figure 2. Measurement flow chart. 


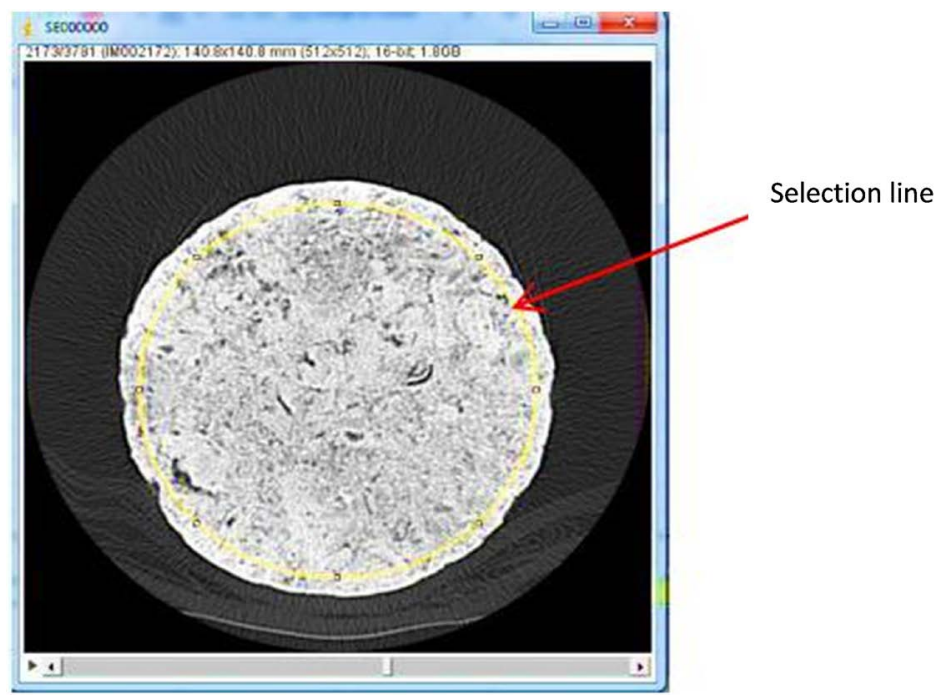

Figure 3. ROI selection of CT image.

Calibrating density is one of the most steps to create the accuracy of this method. In this part, we have to know two of material's density value which is a standard value to calibrate for all pixels in image. With each standard density, we have a correspondent Hounsfield Unit value. So that we make a trend line between $\mathrm{HU}$ values and density values which help to adjust the rest of pixels.

After that, we carry out HU extraction by using Image menu in the software to get $\mathrm{HU}$ values for each cell that intent to the table in Figure 4. Also we extract the histogram of ROI which let us know the max, min, mean values and total cells in our table (Figure 5).

With the result table we got from the software, it is easy to statistics HU values throughout the frequency of appearance and then we cumulate all of values that we received (show in Table 1). If total cumulative value equals total cells, we can use this statistic for porosity calculation.

Based on the table of Hounsfield Unit's common substances which applied to medical grade CT scan in Table 2 [8] [9], we noticed the HU value of air is $-1000 \mathrm{HU}$ and it represent for pore value. So that, the total of cells contain value which equal or less than -1000 is pore volume, the total cells value is represent for bulk volume value.

Then, we can calculate the porosity of single slice by general equation:

$$
\phi=\frac{\text { Pore volume }}{\text { bulk volume }} * 100
$$

or,

$$
\text { Porosity }=\frac{\text { Total cells contain HU value } \leq-1000}{\text { Total cells }} * 100
$$

In accordance with thickness of scan system, we have the quantity of crosssectional slices. The porosity of sample was calculated by average total porosity of all slices. Thus, the more slices we cut the more accuracy we get. 
Table 1. Statistics table for single slice.

\begin{tabular}{ccc}
\hline HU value & Frequency & Cummulative \\
\hline-3000 & 7 & 7 \\
-2999 & 8 & 15 \\
$\ldots$ & $\ldots$ & $\ldots$ \\
-1001 & 278 & 5471 \\
-1000 & 429 & 5900 \\
-999 & 78 & 5978 \\
$\ldots$ & $\ldots$ & $\ldots$ \\
2999 & 41 & 32,155 \\
3000 & 64 & 32,219 \\
\hline
\end{tabular}

Table 2. Hounsfield scale table.

\begin{tabular}{cc}
\hline Tissue & CT Number (HU) \\
\hline Bone & +1000 \\
Liver & $40-60$ \\
Whiter mater & -20 to -30 \\
Grey mater & -37 to -45 \\
Blood & 40 \\
Muscle & $10-40$ \\
Kidney & 30 \\
CSF & 15 \\
Water & 0 \\
Fat & -50 to -100 \\
Air & -1000 \\
\hline
\end{tabular}

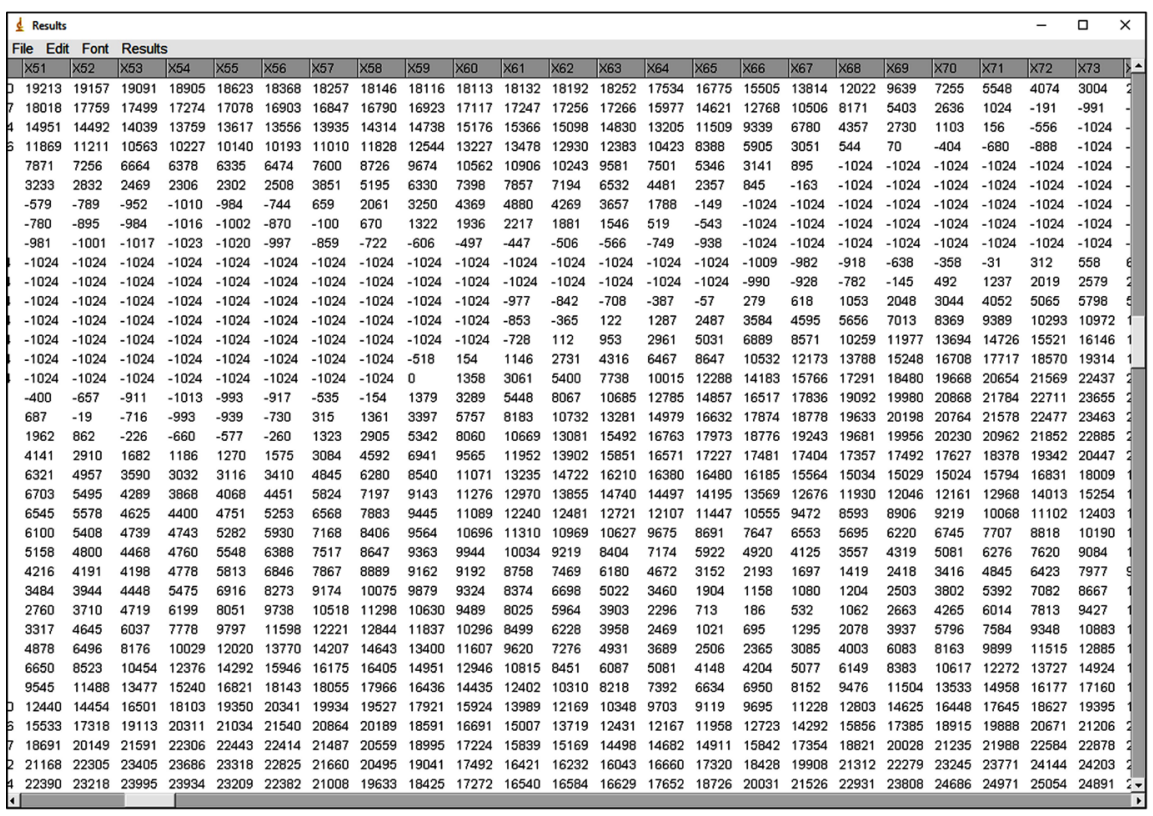

Figure 4. HU values result table. 


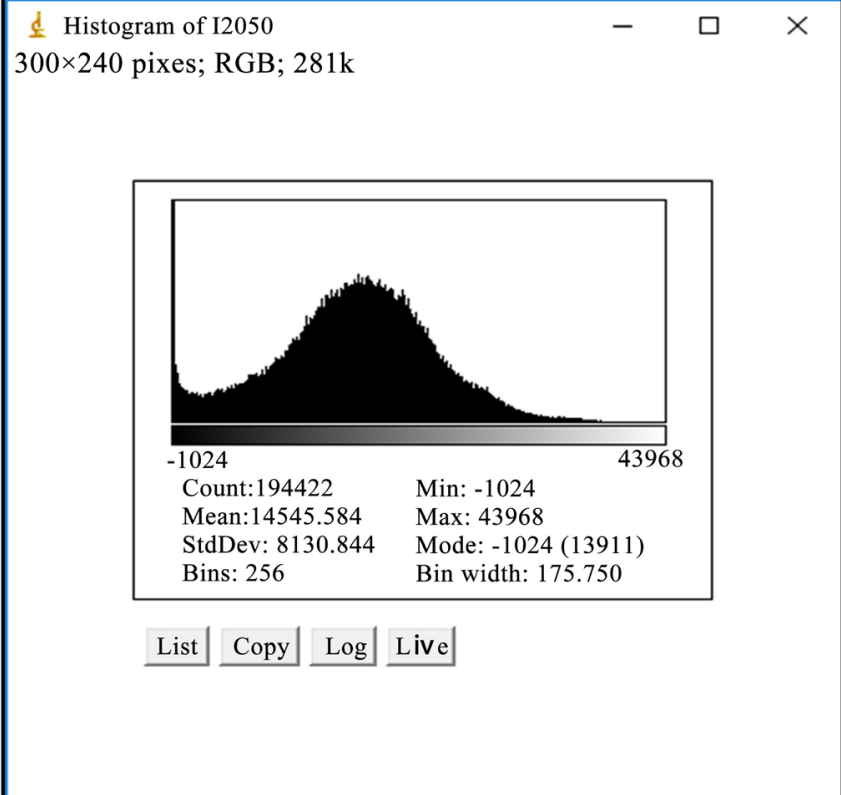

Figure 5. Histogram of HU value in ROI.

\section{Results}

In this research, we collect samples from wells located in Cuu Long basin, Song Hong basin, South Con Son basin and Song Hong Basin to do scan. These basins are main basins in Vietnam and located from North to South of country. The total samples we did are 9 samples where in 5 whole core samples (diameter $=$ 4.0 inches) and 4 conventional core plugs (diameter $=1.5$ inch). They were named from FD1 to FD9. The givens data was showed in Table 3 [10].

Each sample was cut to 10 slices and calculated porosity by follow flow chart's step which describe above (Figure 6).

The result of each sample was showed in Table 4.

During calculate porosity of 9 samples by CT Scan, we also measure porosity of these samples by CMS -300 system which follow Helium injection method to verify the results from CT scanning. Finally, we have a summary table (Table 5).

\section{Conclusion}

The porosity of samples by CT Scan are quite similar with Helium porosity (connective porosity), some minority errors here should be caused by rock type especially isolate porosity in Carbonate rock (FD6).

The goal of this study was to develop non-destructive rock testing procedures using X-ray CT scanning techniques. Traditional rock testing techniques are destructive in nature and may alter the same properties they are designed to measure. Non-destructive tests provide the benefit of not altering the rock structure, so the properties of the unaltered void space may be determined without the risk of introducing systematic errors resulting from rock structure changes during the measurement process. 
Table 3. Given sample data.

\begin{tabular}{cccc}
\hline Sample No. & Porosity, $\%$ & Density, $\mathbf{g} / \mathrm{cm}^{3}$ & Rock type \\
\hline FD1 & 25.20 & 2.70 & Carbonate \\
FD2 & 32.80 & 2.70 & Carbonate \\
FD3 & 15.96 & 2.64 & Sandstone \\
FD4 & 21.70 & 2.63 & Sandstone \\
FD5 & 2.65 & 2.59 & Granite \\
FD6 & 41.70 & 2.71 & Carbonate \\
FD7 & 34.10 & 2.71 & Carbonate \\
FD8 & 28.60 & 2.62 & Sandstone \\
FD9 & 24.50 & 2.63 & Sandstone \\
\hline
\end{tabular}

Table 4. Porosity (\%) result table of each sample.

\begin{tabular}{cccccccccc}
\hline \multirow{2}{*}{ Slice } & \multicolumn{7}{c}{ Sample } \\
\cline { 2 - 9 } & FD1 & FD2 & FD3 & FD4 & FD5 & FD6 & FD7 & FD8 & FD9 \\
\hline S1 & 31.55 & 31.55 & 19.42 & 18.26 & 3.57 & 54.71 & 33.02 & 24.89 & 21.52 \\
S2 & 27.72 & 37.22 & 15.83 & 27.44 & 2.13 & 39.03 & 32.68 & 32.14 & 27.25 \\
S3 & 25.11 & 35.11 & 17.25 & 23.10 & 3.01 & 51.91 & 35.18 & 35.92 & 25.16 \\
S4 & 24.15 & 27.54 & 12.73 & 20.83 & 2.79 & 60.84 & 39.51 & 26.77 & 24.42 \\
S5 & 23.67 & 28.07 & 20.67 & 23.67 & 1.55 & 37.25 & 41.82 & 19.02 & 23.71 \\
S6 & 20.65 & 30.17 & 12.70 & 20.41 & 2.64 & 34.16 & 30.59 & 33.05 & 20.17 \\
S7 & 23.61 & 33.72 & 13.16 & 19.52 & 2.88 & 38.93 & 37.24 & 33.84 & 23.46 \\
S8 & 24.61 & 37.45 & 16.39 & 14.16 & 2.42 & 42.09 & 34.96 & 28.19 & 24.18 \\
S9 & 26.98 & 36.18 & 18.40 & 22.08 & 3.75 & 37.96 & 33.71 & 26.42 & 26.44 \\
S10 & 25.43 & 25.43 & 18.70 & 25.43 & 2.45 & 41.25 & 32.14 & 34.21 & 27.62 \\
Average & 25.35 & $\mathbf{3 2 . 2 4}$ & $\mathbf{1 6 . 5 3}$ & $\mathbf{2 1 . 4 9}$ & $\mathbf{2 . 7 2}$ & $\mathbf{4 3 . 8 1}$ & $\mathbf{3 5 . 0 9}$ & $\mathbf{2 9 . 4 5}$ & $\mathbf{2 4 . 3 9}$ \\
\hline
\end{tabular}

Table 5. Result comparative table.

\begin{tabular}{ccccc}
\hline Sample No. & Porosity by CT Scan & $\begin{array}{c}\text { Porosity by Helium } \\
\text { method }\end{array}$ & Error & Rock type \\
\hline FD1 & 25.35 & 25.20 & $\mathbf{0 . 1 5}$ & Carbonate \\
FD2 & 32.24 & 32.80 & -0.56 & Carbonate \\
FD3 & 16.53 & 15.96 & $\mathbf{0 . 5 7}$ & Sandstone \\
FD4 & 21.49 & 21.70 & $-\mathbf{0 . 2 1}$ & Sandstone \\
FD5 & 2.72 & 2.65 & $\mathbf{0 . 0 7}$ & Granite \\
FD6 & 43.81 & 41.70 & $\mathbf{2 . 1 1}$ & Carbonate \\
FD7 & 35.09 & 34.10 & $\mathbf{0 . 9 8}$ & Carbonate \\
FD8 & 29.45 & 28.60 & $\mathbf{0 . 8 5}$ & Sandstone \\
FD9 & 24.39 & 24.50 & $-\mathbf{0 . 1 1}$ & Sandstone \\
\hline
\end{tabular}




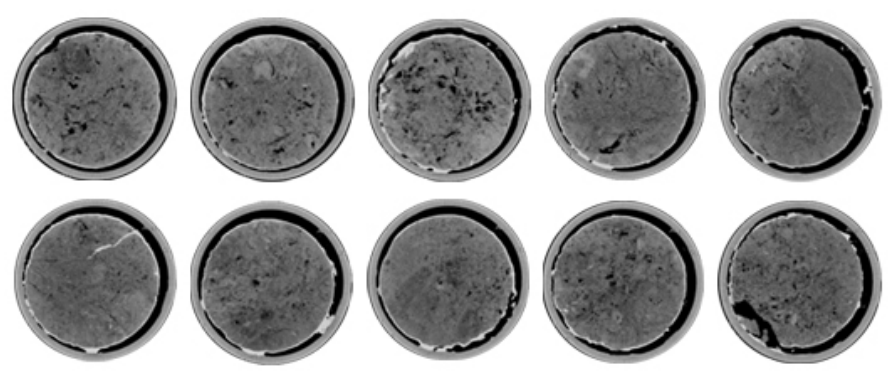

Figure 6. Illustrate 10 slices of sample FD6.

The objective of this research was also to develop image processing techniques to determine rock properties using CT scan images. The porosity results by this technique were compared with results from conventional laboratory. The accuracy of the results is proportional with quantity of slices taken for averaging, especially on heterogeneous samples. Finally, this method can be widely applied in petroleum industry.

\section{Conflicts of Interest}

The authors declare no conflicts of interest regarding the publication of this paper.

\section{References}

[1] Nielsen, B.D. (2004) Non-Destructive Soil Testing Using X-Ray Computed Tomography. Thesis of Master in Science in Civil Engineering, Montana State University, Bozeman, MT.

[2] Gonzalez, R.C. and Woods, R.E. (2007) Digital Image Processing. 3rd Edition, Pearson, London.

[3] Crane, R. (1997) A Simplified Approach to Image Processing. Prentice-Hall, Inc., Upper Saddle River, NJ.

[4] Russ, J.C. (1995) The Image Procesing Handbook. CRC Press, Inc., Boca Raton, FL.

[5] Jain, A.K. (1989) Fundamental of Digital Image Processing. Prentice Hall, Engwood Cliffs.

[6] Shrivakshan, G.T. and Chandrasekar, C. (2012) A Comparison of Various Edge Detection Techniques Used in Image Processing. Bharathiar University, Coimbatore, India.

[7] Paker, J.R. (1997) Algorithms for Image Processing and Computer Vision. John Wiley \& Sons, Inc., Hoboken, NJ.

[8] Low, A. (1991) Introductory Computer Vision and Image Processing. McGraw Hill Book Company (UK) Limited, New York, NY.

[9] Pavlidis, T. (1982) Algorithms for Graphics and Image Processing. Computer Science Press, New York, NY. https://doi.org/10.1007/978-3-642-93208-3

[10] Rourine Core Analysis Report, VPILabs, 2010-2012. 